\title{
Review on the selection of ground motion prescribed by seismic code recommendations
}

\author{
Qingrui Lu \& Shijun Chen ${ }^{1} \&$ Haian Liang ${ }^{2,3,4}$ \\ ${ }^{1}$ Architectual College, East China Institute of Technology, Nanchang 330013, China \\ ${ }^{2}$ Architectual College, East China Institute of Technology, Nanchang 330013, China \\ ${ }^{3}$ School of Civil Engineering ,Guangzhou University ,Guangzhou 510006, China \\ ${ }^{4}$ State Key Laboratory Breeding Base of Nuclear Resources and Environment, East China \\ Institute of Technology, Nanchang, 330013, China
}

KEYWORD: Seismic codes ground motion; Accelerograms

ABSTRACT: The selection of ground motions can be developed on the basis of code provisions, and codes provisions are quite similar worldwide, for example, the main prescription is the compatibility with the design spectrum in a specified range of periods, and the minimum set size is typically from 3-7 records. Here, the provisions of the codes such as FEMA 456, Eurocode 8, and the Italian code NTC 2008 are mainly summarized.

\section{INTRODUCTION}

Geotechnical hazard evaluation requires analysis with respect to some level of shaking, design ground motion is herein adopted to describe a design level of shaking for which satisfactory performance if expected. Design ground motions are often expressed in terms of design spectra, and for sites that could be subjected to shaking from more than one seismic source, development of a design spectra can be complicated, under such circumstances, uniform hazard spectra is allowed, in which spectral ordinates are obtained by individual PSHAs, with proper consideration of all possible seismic source.

Ground motion parameters alone, on many occasions, cannot sufficiently describe the effects of ground shaking, and thus time histories of motion that match design response spectra or uniform hazard spectra within a period range of interest are required. Time histories that can be used are basically of three types: real, artificial and synthetic accelerograms, and the generation or selection of the records to match the reference spectrum has been controversially developed recently ${ }^{[1-3]}$.

The selection of ground motions can be developed on the basis of code provisions, and codes provisions are quite similar worldwide, for example, the main prescription is the compatibility with thedesign spectrum in a specified range of periods, and the minimum set sizeis typically from 3-7 records.Here, the provisions of the codes such as FEMA 456, Eurocode 8, and the Italian code NTC 2008 are mainly summarized ${ }^{[4-6]}$.

\section{Seismic code recommendations}

\section{Eurocode 8}

Eurocode 8 outlines the requirements for the selection of sei]smic input in sec.3.2.3: The seismic motion may be represented in terms of ground acceleration time-histories and depending on the nature of the application and on the information actually available, the description of the seismic motion may be made by using artificial accelerograms, and recorded or simulated accelerograms. For all these three types of accelerograms, the following selection criteria should be satisfied:

- A minimum of 3 accelerograms should be used;

- The mean of the zero period spectral response acceleration values (calculated from the individual time histories) should not be smaller than the value of ag $\mathrm{S}$ for the sit in question;

- In the range of periods between $0.2 \mathrm{~T} 1$ and $2 \mathrm{~T} 1$, where $\mathrm{T} 1$ is the fundamental period of the structure in the direction where the accelerogram will be applied; no value of the mean 5\% damping elastic spectrum, calculated from all time histories, should be less than $90 \%$ of the corresponding value of the $5 \%$ damping elastic response spectrum. 
Specially, artificial accelerograms shall be generated so as to match the elastic response spectra for 5\% viscous damping, and the duration of the accelerograms shall be consistent with the magnitude and other relevant features of the seismic event underlying the establishment of PGA hazard. When site-specific data are not available, for artificial accelerograms, the minimum duration Ts of the stationary part of the accelerograms should be equal to $10 \mathrm{~s}$.

For recorded accelerograms, the samples are required to have the seismogenetic features of the source and to the soilconditions at the site. Records need to be scaled to the peakground acceleration atop of soil layers $(\mathrm{ag} \cdot \mathrm{S})$.

Simulated accelerograms,generated through a physicalsimulation of source and travel path mechanisms, should comply withthe requirements for recorded accelerograms.

Eurocode assigns the spectral shape distinguishing between low and high magnitude events. For surface wave magnitude larger than 5.5, the spectral shape is expressed by:

$$
\begin{aligned}
& 0 \leq T \leq T_{B}: \operatorname{Se}(T)=a_{g} \cdot S \cdot\left[1+\frac{T}{T_{B}} \cdot(\eta \cdot 2.5-1)\right] \\
& T_{B} \leq T \leq T_{C}: \operatorname{Se}(T)=a_{g} \cdot S \cdot \eta \cdot 2.5 \\
& T_{C} \leq T \leq T_{D}: \operatorname{Se}(T)=a_{g} \cdot S \cdot \eta \cdot 2.5\left[\frac{T_{C}}{T}\right] \\
& T_{D} \leq T \leq 4 \mathrm{~s}: \operatorname{Se}(T)=a_{g} \cdot S \cdot \eta \cdot 2.5\left[\frac{T_{C} T_{D}}{T^{2}}\right]
\end{aligned}
$$

Where $T$ is the vibration period of a linear SDOF; $a_{g}$ is the design ground acceleration on type A site class; $S$ is the soil factor; $T_{B}{ }_{C} T_{C}$ are the limiting periods of the spectrum's plateau; ${ }_{D}^{P}$ is the lowest period of the constant spectral portion; $\eta$ is the damping correction factor, and it is equal to one for $5 \%$ viscous damping.

The spectral ordinates and shapes depend on the seismic hazard level as well as site class. The five site classes, based on the shear-wave velocity in the upper $30 \mathrm{~m}$ range, are summarized in Table 1. The controlling parameters describing the elastic response spectra are shown in Table 2, and the resulting curves can be plotted as figure 1 .

Table 1 . Site classification based on the shear-wave velocity.

\begin{tabular}{lc}
\hline $\begin{array}{l}\text { Site class } \\
\text { A-Rock or other rock-like } \\
\text { geological formation }\end{array}$ & $\mathrm{Vs}_{30}(\mathrm{~m} / \mathrm{s})$ \\
$\begin{array}{l}\text { B-Deposits of very dense } \\
\text { sand, gravel, or very stiff } \\
\text { clay (stiff soil) }\end{array}$ & $>800$ \\
$\begin{array}{l}\text { C-Deep deposits of dense or } \\
\text { medium-dense sand, gravel } \\
\text { or stiff clay (soft soil) }\end{array}$ & $180-360$ \\
$\begin{array}{l}\text { D-Deposits of loose-to- } \\
\text { medium cohesionless } \\
\text { soil(very soft soil) }\end{array}$ & $<180$ \\
$\begin{array}{l}\text { E-A soil profile consisting of } \\
\text { a surface alluvium layer } \\
\text { (Alluvional) }\end{array}$ & $\begin{array}{c}\text { Vs values of type C or D and } \\
\text { thickness varying between } \\
\text { about } 5 \text { m and } 20 \mathrm{~m},\end{array}$ \\
& $\begin{array}{c}\text { underlain by stiffer material } \\
\text { with Vs }>800 \mathrm{~m} / \mathrm{s}\end{array}$ \\
\hline
\end{tabular}


Table 2. The controlling parameters of the elastic response spectra.

\begin{tabular}{ccccc}
\hline Site class & S-factor & TB $(\mathrm{s})$ & TC $(\mathrm{s})$ & TD(s) \\
A & 1 & 0.15 & 0.4 & 2.0 \\
B & 1.2 & 0.15 & 0.5 & 2.0 \\
C & 1.15 & 0.2 & 0.6 & 2.0 \\
D & 1.35 & 0.2 & 0.8 & 2.0 \\
E & 1.4 & 0.15 & 0.5 & 2.0 \\
\hline
\end{tabular}

It may be argued that the code spectrum represents a crude approximation of the uniform hazard spectrum, because the connection between the code spectrum and the seismic hazard is set anchoring the spectra to $\mathrm{a}_{\mathrm{g}}$ values with a certain exceedance probability.

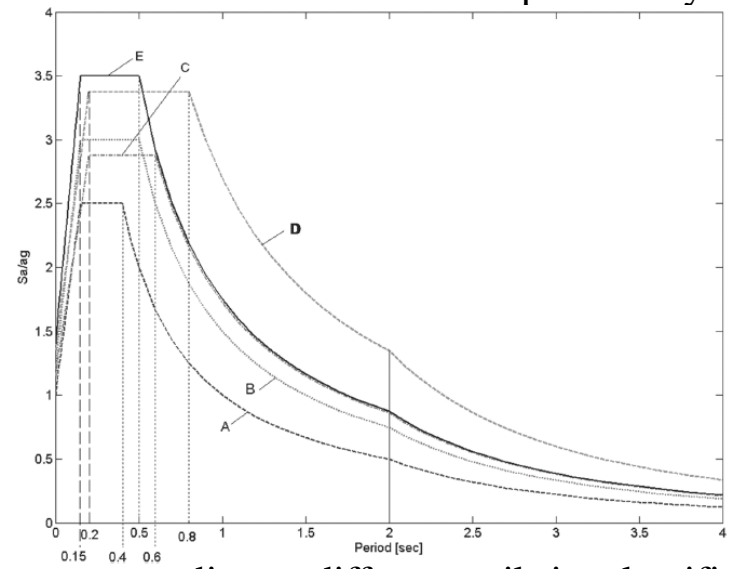

Figure 1 The design spectrum corresponding to different soil site classifications.

It may be argued that the code spectrum represents a crude approximation of the uniform hazard spectrum, because the connection between the code spectrum and the seismic hazard is set anchoring the spectra to $a_{g}$ values with a certain exceedance probability.

\section{Italian seismic code(NTC, 2008)}

The NTC outlines the requirements for the seismic input for dynamic analysis in section3.2.3.6, after specifying the elastic response spectrum. The signals that can be used for theseismic structural analysis can belong to the following three categories: artificial waveforms,simulated accelerograms, and natural records from real events. Artificial records should haveduration of at least 10 seconds in their pseudo-stationary part, and they cannot beused in the assessment of geotechnical structures. Synthetic generated by simulationof earthquake rupture and propagation process should refer to a characteristicscenario for the site in terms of magnitude, distance and other source seismologicalcharacteristics; finally, real records should reflect the event dominating thehazard at the site.

The main condition to be satisfiedby artificial records is that the average elastic spectrum (of the chosen set) does not underestimatethe 5\% damping elastic code spectrum, with a $10 \%$ tolerance, in the larger range of periodsbetween $[0.15 \mathrm{~s}, 2 \mathrm{~s}]$ and $[0.15 \mathrm{~s}, 2 \mathrm{~T} 1]$ for safety checks at ultimate limit state (T1 is thefundamental period of the structure in the direction where the accelerograms will be applied) orin the larger period ranges between [0.15 s, $2 \mathrm{~s}$ ] and [0.15 s, $1.5 \mathrm{~T} 1]$, for structural safety checksat serviceability limit states. For seismically isolated structures, the code provides a narrowerrange of matching around the fundamental period, [0.15 s, 1.2 Tis], where Tis is the equivalentperiod of the isolated structure.

Natural accelerograms or accelerograms generated through a physical simulation of sourcemechanism, travel, and path, may be used, provided that the samples used are adequatelyqualified with regard to the seismogenic features of the source and the soil conditions appropriateto the site. Selected real records have to be scaled to match the elastic response spectrum in arange of periods of interest for the shaking of the structure.

NTC 2008 describes the spectral shape distinguishing between horizontal and vertical components of accelerograms. Fort the horizontal components, the elastic spectral shape is expressed by: 


$$
\begin{aligned}
& 0 \leq T \leq T_{B}: \operatorname{Se}(T)=a_{g} \cdot S \cdot \eta \cdot F_{0} \cdot\left[\frac{T}{T_{B}}+\frac{1}{\eta \cdot F_{0}}\left(1-\frac{T}{T_{B}}\right)\right] \\
& T_{B} \leq T \leq T_{C}: \operatorname{Se}(T)=a_{g} \cdot S \cdot \eta \cdot F_{0} \\
& T_{C} \leq T \leq T_{D}: \operatorname{Se}(T)=a_{g} \cdot S \cdot \eta \cdot F_{0} \cdot\left[\frac{T_{C}}{T}\right] \\
& T_{D} \leq T \leq 4 \mathrm{~s}: \operatorname{Se}(T)=a_{g} \cdot S \cdot \eta \cdot F_{0} \cdot\left[\frac{T_{C} T_{D}}{T^{2}}\right]
\end{aligned}
$$

For the vertical components, the elastic spectral shape is expressed by:

$0 \leq T \leq T_{B}: \operatorname{Sve}(T)=a_{g} \cdot S \cdot \eta \cdot F_{v} \cdot\left[\frac{T}{T_{B}}+\frac{1}{\eta \cdot F_{v}}\left(1-\frac{T}{T_{B}}\right)\right]$

$T_{B} \leq T \leq T_{C}: \operatorname{Sve}(T)=a_{g} \cdot S \cdot \eta \cdot F_{v}$

$T_{C} \leq T \leq T_{D}: \operatorname{Sve}(T)=a_{g} \cdot S \cdot \eta \cdot F_{v} \cdot\left[\frac{T_{C}}{T}\right]$

$T_{D} \leq T \leq 4 \mathrm{~s}: \operatorname{Sve}(T)=a_{g} \cdot S \cdot \eta \cdot F_{v} \cdot\left[\frac{T_{C} T_{D}}{T^{2}}\right]$

\section{The Standards New Zealand (2004a) ${ }^{[7]}$}

The standard states in section 5.5.1 that: "The ground motion records shall be selected from actual records that have aseismological signature (i.e. magnitude, source characteristic (including faultmechanism) and source-to-site distance) the same as (or reasonably consistent with)the signature of the events that significantly contributed to the target design spectra ofthe site over the period range of interest. The ground motion is to have been recordedby an instrument located at a site, the soil conditions of which are the same as (orreasonably consistent with) the soil conditions at the site."

\section{FEMA 45}

For both 2D analysis and 3D analysis, the following requirements in FEMA 450 should be matched:

- Appropriate acceleration histories shall be obtained from records of events having magnitudes, fault distance and source mechanisms that are consistent with those that control the maximum considered earthquake.

- A suite of not fewer than 3 appropriate ground motions shall be used in the analysis.

- Where the required number of appropriate recorded ground motion records are not available, appropriate simulated ground motion records shall be used to make up the total number required.

For 2D analysis, it is required that:

- Each ground motion shall consist of a horizontal acceleration history selected from an actual recorded event

- The ground motions shall be scaled such that for each period between $0.2 \mathrm{~T}$ and $1.5 \mathrm{~T}$, the average of the five-percent-damped response spectra for the suite of motions is not less than the corresponding ordinate of the design response spectrum.

For 3D analysis, on the other hand, certain provisions should be satisfied as following: 
- Ground motions shall consist of pairs of appropriate horizontal ground motion acceleration components that shall be selected and scaled from individual recorded events

- For each pair of horizontal ground motion components, an SRSS spectrum shall be constructed by taking the square-root-of-the-sum-of-the-squares(SRSS) of the five-percentdamped response spectra for the components (where an identical scale factor is applied to both components of a pair)

- Each pair of motions shall be scaled such that for each period between 0.2T and 1.5 T the average of the SRSS spectra from all horizontal component pairs is not less than 1.3 times the corresponding ordinate of the design response spectrum.

In addition, some specific suggestions for input selections of accelerograms to be used in analyses are also provided, for example, in Part3, Section 3.4, several acceleration time-histories of rock motions, typically at least four, should be selected for site response analysis. These acceleration time-histories should be selected after evaluating the types of earthquakes sources, magnitudes, and distances that predominantly contribute to the seismic hazard at the site.

According to the above code provisions, the following selection criteria can be concluded:

- For accelerogram types, artificial accelerograms are accepted in Eurocode 8, while NEHRP 2003 gives preference to recordedaccelerograms;

- Compatibility between selected time-histories and target response spectrum should be satisfied;

- Due to the probabilistic nature of seismic action, a number of records, with the minimum number from 3 to 7 , are required;

- For the period range of interest, the lower limit $(0.2 \mathrm{~T})$ accounts for higher modes of vibration, while the upper limit (1.5-2.0T) accounts for 'softening' of the structure due to inelastic response.

There are also certain limitations in the above codes, for example, the duration of the records are not directly mentioned in the code provisions, on the other hand, these codes take into account site effects by lumping groups of similar soil profiles together so that their provisions apply to broad ranges of soil conditions, within which the local conditions of a particular site are expected to fall, thus the accelerograms developed from code provisions are usually conservative.

\section{CONCLUSIONS}

The provisions of the codes such as FEMA 456, Eurocode 8, and the Italian code NTC 2008 are mainly summarized. Codes provisions are quite similar worldwide, for example, the main prescription is the compatibility with the design spectrum in a specified range of periods, and the minimum set size is typically from 3-7 records. There are also certain limitations in the codes, that is, he accelerograms developed from code provisions are usually conservative.

\section{References}

1 Aki, K., Richards, P.G. (1980): Quantitative seismology. Theory and Methods, vols. I, II (W. H. Freemanand Company, San Francisco, USA 1980), 948 pp.

2 Kramer, S.L. (1996): Geotechnical earthquake engineering. Prentice Hall, Upper Saddle River, NJ.

3 Reiter, L. (1990): Earthquake hazard analysis - issues and insights. Columbia University Press, New York, $254 \mathrm{p}$.

4 Eurocode 8. (2003):Design for structures for earthquakes resistance. Part 1: General rules, seismic actions and rules for buildings. Final Draft—pren1998-1. December 2003 edition.

5 Nuove Norme Tecniche per le Costruzioni. (2008): Gazzetta Ufficiale n. 29 del 4 febbraio 2008 - Suppl. Ordinario n. 30, Consiglio Superiore dei Lavori Pubblici, Italy.

6 FEMA.(1999): HAZUS99 - Earthquake Loss Estimation Methodology: User's Manual, Federal Emergency Management Agency, Washington, DC. 
International Forum on Energy, Environment Science and Materials (IFEESM 2015)

7 Earthquake Actions (New Zealand). Committee draft DR PPCD 8, Standards New Zealand, Wellington. 